Check for updates

Cite this: Phys. Chem. Chem. Phys., 2019, 21, 14766

Received 22nd March 2019 Accepted 21st May 2019

DOI: $10.1039 / c 9 c p 01618 j$

rsc.li/pccp

\title{
Structures, dipole moments and excited state lifetime of isolated 4-cyanoindole in its ground and lowest electronically excited singlet states $\dagger$
}

\author{
Marie-Luise Hebestreit, (D) Michael Schneider, ${ }^{a}$ Hilda Lartian, ${ }^{a}$ Vivienne Betz, ${ }^{a}$ \\ Michael Heinrich, ${ }^{a}$ Mirko Lindic, (D) ${ }^{a}$ Myong Yong Choi $\mathbb{D}^{\mathrm{b}}$ and Michael Schmitt (D) *a
}

\begin{abstract}
The rotationally resolved electronic spectrum of 4-cyanoindole and some N-D and C-D deuterated isotopologues has been measured and analyzed. Dipole moments in the ground and electronically excited state have been determined, using electronic Stark spectroscopy. From the geometry changes upon excitation, orientation of the transition dipole moment, and the values for the permanent dipole moments, the lowest excited singlet state could be shown to be of $L_{a}$ symmetry. The excited state lifetime of isolated 4-cyanoindole has been determined to be $11 \mathrm{~ns}$, while for the ringdeuterated isotopologues lifetimes between 5 and 6 ns have been found. The different behavior of 3-, 4-, and 5-cyanoindole is

discussed on the basis of the different electronic nature of the electronically excited singlet states.
\end{abstract}

\section{Introduction}

It is a well-accepted paradigm, that nature selects chromophores as building blocks, which efficiently dissipate the absorbed photon energy due to their short excited state lifetime. This lifetime strongly depends on the direct molecular surrounding and is therefore strongly different for isolated molecules in the gas phase and in solution of different solvents. ${ }^{1-3}$

The radiative lifetime of isolated molecules can be calculated from the Lorentz contribution to the total linewidth of the transitions in rovibronically resolved fluorescence spectra. For isolated molecules this lifetime depends on the fluorescence rate constant $k_{\mathrm{F}}$ and the coupling to the dense manifold of dark background states, which carry no oscillator strengths. For background states of the same multiplicity we deal with internal conversion (IC), while different multiplicities give rise to intersystem crossing (ISC). Thus, the energetic position of the (dark) triplet manifold with respect to the initially excited (bright) singlet state determines the ISC rate $k_{\mathrm{ISC}}$. The fluorescence lifetime taking IC and ISC into account is $\tau=1 /\left(k_{\mathrm{F}}+k_{\mathrm{IC}}+k_{\mathrm{ISC}}\right)$. When talking about IC, conical intersections (CIs) of the primarily photo-excited state with other states of proper symmetry, have to be considered. ${ }^{4}$ These conical intersections are by no way rare events, as stated by Truhlar and Mead: “(...) if one encounters a very small

\footnotetext{
${ }^{a}$ Heinrich-Heine-Universität, Institut für Physikalische Chemie I, D-40225 Düsseldorf, Germany. E-mail: mschmitt@uni-duesseldorf.de;

Fax: +49 2118113689 ; Tel: +492118112100

${ }^{b}$ Department of Chemistry (BK21+) and Research Institute of Natural Science, Gyeongsang National University, Jinju 52828, Republic of Korea

$\dagger$ Electronic supplementary information (ESI) available. See DOI: 10.1039/c9cp01618j
}

electronic energy gap along a path through configuration space, it is much more likely to be associated with the neighborhood of a conical intersection than with an avoided intersection." ${ }^{, 5}$ Even if the primarily excited electronic state is not directly linked to the ground state through a single CI, a sequence of CIs, involving one repelling state, which is coupled to the ground state has strong effects of the lifetime of the primarily excited state. Its lifetime is then strongly affected by the relative positions of these CIs. ${ }^{2,6-8}$ Even well-separated $\pi \pi^{*}$ states show CIs, that affect their photophysical properties..$^{9-13}$

Upon solvation, a plethora of nonradiative channels open, which are closed for the isolated molecule. In most cases, this leads to drastically shorter lifetimes in solution. The experiment of choice in solution is time-correlated single photon counting (TCSPC).

A special class of indole derivatives, the cyanotryptophans are used as dual infrared and fluorescence spectroscopic labels to assess structural dynamics in proteins. ${ }^{14}$ Markiewicz et al. have shown that 5-cyanotryptophan fluorescence can be utilized as a sensitive probe of protein hydration. ${ }^{15}$

The fluorescence lifetime of the different cyanoindoles in solution has been found to depend considerably on the solvent. ${ }^{16,17}$ The reason for these differing photophysical properties, depending on the position of the cyano substituent is due to the relative energetic positions of the lowest excited $\pi \pi^{*}$ singlet states. These states can be classified in the nomenclature of Platt for cata-condensed aromatics as ${ }^{1} \mathrm{~L}_{\mathrm{a}}$ and ${ }^{1} \mathrm{~L}_{\mathrm{b}}$ states ${ }^{18}$ a scheme, which was later extended to indole by Weber. ${ }^{19}$

Experimental evidence for the relative position of the ${ }^{1} \mathrm{~L}_{\mathrm{a}}$ and ${ }^{1} \mathrm{~L}_{\mathrm{b}}$ states in the indole chromophore has been collected 
for decades. ${ }^{20-28}$ Also theory provided important details for the classification of these states. ${ }^{7,10,11,29-32}$ Domcke and Sobolewski have shown that the photophysics of indoles can be influenced by the existence of a $\pi \sigma^{*}$ state, which is dissociative along the NH coordinate. ${ }^{2,6,7,33}$

Much less fundamental investigations on the ${ }^{1} \mathrm{~L}_{\mathrm{a}}$ and ${ }^{1} \mathrm{~L}_{\mathrm{b}}$ states has been performed for the cyanoindoles. Rotationally resolved electronic spectra of 5-cyanoindole have been presented. ${ }^{34-36}$ From the comparison of the experimental findings to quantum chemical calculations at the coupled cluster level of theory, the lowest excited singlet state could be shown to be the ${ }^{1} \mathrm{~L}_{\mathrm{a}}$ in contrast to most other indole derivatives, which have the ${ }^{1} \mathrm{~L}_{\mathrm{b}}$ state as lowest excited state. 5-Cyanoindole $\left(\mathrm{H}_{2} \mathrm{O}\right)_{n}$ with $n=0-2$ has been investigated using UV-UV hole burning and IR-dip spectroscopy by Min et $a l^{37}$ They found a different photodissociation pattern of the 5-cyanoindole-water clusters compared to the respective 3-cyanoindole-water clusters. ${ }^{38,39}$ Using TD-DFT with the LC-BLYP, M06-2X, and B3LYP functionals, the same energetic order of the lowest $\pi \pi^{*}$ states was found compared to calculations using the second-order approximate coupled cluster singles and doubles model (CC2). ${ }^{34}$

In 3-cyanoindole, the order of the ${ }^{1} \mathrm{~L}_{\mathrm{a}}$ and ${ }^{1} \mathrm{~L}_{\mathrm{b}}$ states is reversed again, the ${ }^{1} \mathrm{~L}_{\mathrm{b}}$ state being the lower one as determined by rotationally resolved electronic Stark spectroscopy. ${ }^{40}$ The water clusters of 3-cyanoindole $\left(\mathrm{H}_{2} \mathrm{O}\right)_{n}$ with $n=1,2$ have been studied in the group of Choi by UV-UV hole burning and IR-dip spectroscopy. ${ }^{38,39}$ Based on the comparison of the IR-dip spectra with the results of normal mode analyses and a Franck-Condon analysis of the mass-selected R2P spectra of 3-cyanoindole $\left(\mathrm{H}_{2} \mathrm{O}\right)_{n}$ with $n=1,2$ they deduced a linearly bound water moiety with an $\mathrm{N}-\mathrm{H} \cdots \mathrm{OH}_{2}$ binding motif. This structure was confirmed by the rotational constants from rotationally resolved electronic spectroscopy. ${ }^{40}$ The excited state lifetime of 3-cyanoindole has been determined to be $9.8 \mathrm{~ns}$, while that of the 3-cyanoindole $\left(\mathrm{H}_{2} \mathrm{O}\right)_{1}$ cluster shows a considerably shorter lifetime of 3.6 ns. Interestingly, the fluorescence lifetime of 3-cyanoindole in water solution is the shortest of all cyanoindoles. ${ }^{16}$

Other 4-substituted indoles show a different behavior concerning their lifetimes, compared to indole. 4-Hydroxyindole has an unusually short excited state lifetime of $200 \mathrm{ps}^{41}$ Also 4-methoxyindole (5.0 ns) and 4-fluoroindole (7.4 ns) exhibit shorter lifetimes than indole (17.6 ns) and other monosubstituted indoles, whose lifetimes lie in a range of $10-18 \mathrm{~ns} .{ }^{41}$ In general, the lifetime of the deuterated compounds is higher. For monodeuterated 4-hydroxyindole (deuteration at the $\mathrm{OH}$ site) an increase of more than one order of magnitudes is observed $(4.3 \mathrm{~ns}) .{ }^{41}$ In contrast, the lifetime of 4-methylindole $(16.2 \mathrm{~ns})$ is close to the indole value. ${ }^{41}$

In the present study, we investigate the electronic nature of the lowest excited state of 4-cyanoindole and some deuterated species, their excited state lifetimes and dipole moments in both ground and excited singlet states, using a combination of rotationally resolved fluorescence spectroscopy, rotationally resolved electronic Stark spectroscopy, time-correlated single photon counting, and $a b$ initio quantum chemical calculations for a deeper understanding of the photophysics of electronically excited cyanoindoles.

\section{Experimental section}

\subsection{Experimental procedures}

2.1.1 Rotationally resolved electronic (Stark) spectroscopy. 4-Cyanoindole ( $>98 \%$ ) was purchased from Activate Scientific and used without further purification. To record rotationally resolved electronic spectra, the sample was heated to $150{ }^{\circ} \mathrm{C}$ and co-expanded with 300 mbar of argon into the vacuum through a $147 \mu \mathrm{m}$ nozzle. After the expansion, a molecular beam was formed using two skimmers ( $1 \mathrm{~mm}$ and $3 \mathrm{~mm}$ ) linearly aligned inside a differentially pumped vacuum system consisting of three vacuum chambers. The molecular beam was crossed at right angles with the laser beam $360 \mathrm{~mm}$ downstream of the nozzle. To create the excitation beam, $10 \mathrm{~W}$ of the $532 \mathrm{~nm}$ line of a diode pumped solid state laser (Spectra-Physics Millennia eV) pumped a single frequency ring dye laser (Sirah Matisse DS) operated with rhodamine 6G. The fluorescence light of the dye laser was frequency doubled in an external folded ring cavity (Spectra Physics Wavetrain) with a resulting power of about 1-2 $\mathrm{mW}$ during the experiments. The fluorescence light of the samples was collected perpendicular to the plane defined by laser and molecular beam by an imaging optics setup consisting of a concave mirror and two planoconvex lenses onto the photocathode of a UV enhanced photomultiplier tube (Thorn EMI 9863QB). The signal output was then discriminated and digitized by a photon counter and transmitted to a PC for data recording and processing. The relative frequency was determined using a quasi confocal Fabry-Perot interferometer. The absolute frequency was obtained by comparing the recorded spectrum to the tabulated lines in the iodine absorption spectrum. ${ }^{42}$ A detailed description of the experimental setup for rotationally resolved laser induced fluorescence spectroscopy has been given previously. ${ }^{43,44}$ The Stark plates consist of a parallel pair of electro-formed nickel wire grids (18 mesh per $\mathrm{mm}, 50 \mathrm{~mm}$ diameter) with a transmission of $95 \%$ in the UV. Their effective distance is $23.49 \pm 0.05 \mathrm{~mm}$, symmetrically aligned with respect to the laser beam. This distance was calibrated using the accurately known dipole moment of benzonitrile. ${ }^{45,46}$ The polarization plane of the incoming laser beam can be rotated by $90^{\circ}$ inside the vacuum chamber by means of an achromatic $\lambda / 2$ plate (Bernhard Halle $240-380 \mathrm{~nm}$ ). The $\lambda / 2$ plate can be pushed in or pulled out of the laser beam using a linear motion vacuum feedthrough. By changing the plane of polarization one can choose between a parallel set-up (selection rules $\Delta M=0$ ) and perpendicular (selection rules $\Delta M= \pm 1$ ).

2.1.2 Time-correlated single photon counting. TCSPC was performed with a DeltaFlex Ultima spectrometer (HORIBA Jobin Yvon), equipped with a supercontinuum light source SuperK Extreme EXR-20 and frequency doubler SuperK Extend-UV/DUV (both NKT Photonics). The signal was recorded under magic angle conditions, a pulse repetition rate of $19.2 \mathrm{MHz}$ and a sample temperature of $20^{\circ}$. The instrument response function (IRF, FWHM approximately $60 \mathrm{ps)} \mathrm{was} \mathrm{recorded,} \mathrm{using} \mathrm{a} \mathrm{scattering}$ solution (Ludox) for each excitation wavelength and used in the fits applying an iterative reconvolution algorithm to minimize $\chi^{2}$. All solvents used have been dehydrated and were stored and used under inert gas (Ar). 


\subsection{Quantum chemical calculations}

Structure optimizations were performed employing a Dunning's correlation-consistent polarized valence triple zeta (cc-pVTZ) basis set from the Turbomole library. ${ }^{47,48}$ The equilibrium geometries of the electronic ground and the lowest excited singlet states were optimized using the approximate coupled cluster singles and doubles model (CC2) employing the resolution-ofthe-identity (RI) approximation. ${ }^{49-51}$ For the structure optimizations spin-component scaling (SCS) modifications to CC2 were taken into account. ${ }^{52}$ Vibrational frequencies and zero-point corrections to the adiabatic excitation energies were obtained from numerical second derivatives using the NumForce script. ${ }^{53}$

\subsection{Fits of the rovibronic spectra using evolutionary algorithms}

The evaluation of the molecular parameters in the Hamiltonian is performed in an automated process based on evolutionary algorithms. ${ }^{54-57}$ They provide a straightforward approach to find the global minimum in a multi-parameter optimization. Here, we apply the covariance matrix adaptation evolution strategy (CMA-ES), which is described in detail elsewhere. ${ }^{58,59}$

\section{Results}

\subsection{Computational results}

The CC2/cc-pVTZ optimized structure of 4-cyanoindole in the ground and lowest excited singlet state is planar in both states. The Cartesian coordinates of all optimized states are given in the ESI. $\dagger$ Contrary to 5-cyanoindole, generic CC2 and its spin component scaled variant SCS-CC2 yield the same state as lowest excited singlet state. ${ }^{34}$ The lowest excited singlet state for both CC2 and SCS-CC2 is the ${ }^{1} \mathrm{~L}_{\mathrm{a}}$ state, what can be inferred from the calculated transition dipole moment orientation (cf. Table 1 and Fig. 1) and the orbital contributions to the excitation, given in Fig. S7 of the ESI. $\uparrow$ The lowest singlet state is reached by an almost pure LUMO $\leftarrow$ HOMO transition, an indication for an ${ }^{1} \mathrm{~L}_{\mathrm{a}}$ state, while the adiabatically second state is composed of LUMO $\leftarrow$ HOMO-1 and LUMO $+1 \leftarrow$ HOMO contributions typical for an ${ }^{1} \mathrm{~L}_{\mathrm{b}}$ state.

The molecular parameters (rotational constants $A, B$, and $C$ in both electronic states, the inertial defects $\Delta I$, the angle $\theta$ of the transition dipole moment with the inertial $a$ axis, the angle $\theta_{\mathrm{D}}$ of the permanent dipole moment with the inertial $a$-axis, and the zero-point corrected origin frequency $\nu_{0}$ ) for all isotopologues studied, are compiled in Table 1 and are compared to the respective experimental results, which are described in detail in Section 3.2.

Table 2 gives the relative vertical energies of the lowest $\pi \pi^{*}$ and $\pi \sigma^{*}$ states at the ground state geometry of 4-cyanoindole, along with their oscillator strengths and the dipole moments of the respective state. We included the excitation energies of indole, 3-cyanoindole and 5-cyanoindole for comparison. Interestingly, at the ground state geometry, the $L_{b}$ state is always the $S_{1}$, while adiabatically it changes to the $\mathrm{S}_{2}$ for 4 - and 5-cyanoindole.

Hougen and Watson ${ }^{60}$ have shown that a molecule of low enough symmetry, which undergoes an electronic transition,

Table 1 SCS-CC2/cc-pVTZ computed and experimental molecular parameters of 4-cyanoindole (4-Cl) and the 1-, 2- and 3-deuterated isotopologue $\left(\mathrm{d}_{1}\right)$. Doubly primed parameters belong to the electronic ground and single primed to the excited state. $\theta_{\mathrm{D}}$ is the angle of the permanent dipole moment vector with the main inertial a-axis. A negative sign of this angle means an anti-clockwise rotation of the main inertial $a$-axis onto the dipole moment vector, shown in Fig. 1. $\theta$ is the angle of the transition dipole moment vector with the main inertial $a$-axis. The same convention for its sign is used as for $\theta_{\mathrm{D}}$. For details see text

\begin{tabular}{|c|c|c|c|c|c|c|c|c|}
\hline & \multicolumn{4}{|c|}{ Theory SCS-CC2 } & \multicolumn{4}{|l|}{ Experiment } \\
\hline & 4 -CI & $4-\mathrm{CI}\left(1-\mathrm{d}_{1}\right)$ & $4-\mathrm{CI}\left(2-\mathrm{d}_{1}\right)$ & $4-\mathrm{CI}\left(3-\mathrm{d}_{1}\right)$ & 4 -CI & $4-\mathrm{CI}\left(1-\mathrm{d}_{1}\right)$ & $4-\mathrm{CI}\left(2-\mathrm{d}_{1}\right)$ & $4-\mathrm{CI}\left(3-\mathrm{d}_{1}\right)$ \\
\hline$A^{\prime \prime} / \mathrm{MHz}$ & 1790.15 & 1789.75 & 1748.49 & 1746.22 & 1795.11(3) & 1791.56(3) & 1747.85(2) & $1748.40(4)$ \\
\hline$B^{\prime \prime} / \mathrm{MHz}$ & 1138.03 & 1110.06 & 1120.49 & 1137.99 & $1146.83(2)$ & $1116.74(2)$ & $1116.90(1)$ & $1145.11(3)$ \\
\hline$C^{\prime \prime} / \mathrm{MHz}$ & 695.74 & 685.12 & 682.88 & 688.99 & $699.79(1)$ & $687.96(2)$ & $681.52(1)$ & $691.89(2)$ \\
\hline$\Delta I^{\prime \prime} / \mathrm{amu} \AA^{2}$ & 0.00 & 0.00 & 0.00 & 0.00 & -0.02 & -0.03 & -0.07 & 0.04 \\
\hline$\mu_{\mathrm{a}}{ }^{\prime \prime} / \mathrm{D}$ & \pm 6.13 & - & - & - & $\pm 6.08(1)$ & - & - & - \\
\hline$\mu_{\mathrm{b}}^{\prime \prime} / \mathrm{D}$ & \pm 1.82 & - & - & - & $\pm 1.68(1)$ & - & - & - \\
\hline$\mu^{\prime \prime} / \mathrm{D}$ & 6.39 & - & - & - & $6.31(1)$ & - & - & - \\
\hline$\theta_{\mathrm{D}}{ }^{\prime \prime} /^{\circ}$ & -16.5 & - & - & - & -15.45 & - & - & - \\
\hline$A^{\prime} / \mathrm{MHz}$ & 1769.07 & 1768.29 & 1726.20 & 1727.76 & $1778.80(4)$ & $1774.93(4)$ & $1733.54(3)$ & $1734.43(5)$ \\
\hline$B^{\prime} / \mathrm{MHz}$ & 1123.71 & 1096.29 & 1107.20 & 1123.59 & 1131.14(3) & 1101.72(3) & 1101.71(3) & $1129.24(4)$ \\
\hline$C^{\prime} / \mathrm{MHz}$ & 687.20 & 676.73 & 674.54 & 680.83 & $691.51(2)$ & $679.85(3)$ & $673.71(2)$ & $683.95(3)$ \\
\hline$\Delta I^{\prime} / \mathrm{amu} \AA^{2}$ & 0.00 & 0.00 & 0.00 & 0.00 & -0.06 & -0.09 & -0.11 & -0.01 \\
\hline$\mu_{\mathrm{a}}^{\prime} / \mathrm{D}$ & \pm 9.42 & - & - & - & $\pm 8.92(1)$ & - & - & - \\
\hline$\mu_{\mathrm{b}}{ }^{\prime} / \mathrm{D}$ & \pm 0.10 & - & - & - & $\pm 0.29(3)$ & - & - & - \\
\hline$\mu^{\prime} / \mathrm{D}$ & 9.42 & - & - & - & $8.92(1)$ & - & - & - \\
\hline$\theta_{\mathrm{D}}^{\prime} /^{\circ}$ & 0.60 & - & - & - & 1.86 & - & - & - \\
\hline$\Delta A / \mathrm{MHz}$ & -21.08 & -21.46 & -22.29 & -18.46 & $-16.31(1)$ & $-16.63(1)$ & $-14.35(1)$ & $-13.98(1)$ \\
\hline$\Delta B / \mathrm{MHz}$ & -14.32 & -13.77 & -13.29 & -14.40 & $-15.69(1)$ & $-15.02(1)$ & $-15.20(1)$ & $-15.87(1)$ \\
\hline$\Delta C / \mathrm{MHz}$ & -8.45 & -8.39 & -8.34 & -8.16 & $-8.28(1)$ & $-8.11(1)$ & $-7.81(1)$ & $-7.94(1)$ \\
\hline$\Delta \nu_{\text {Gauss }} / \mathrm{MHz}$ & - & - & - & - & 15.17 & - & - & - \\
\hline$\Delta \nu_{\text {Lorentz }} / \mathrm{MHz}$ & - & - & - & - & $14.41(2)$ & $31.70(3)$ & $25.72(1)$ & $27.23(1)$ \\
\hline$\tau / \mathrm{ns}$ & - & - & - & - & $11.04(3)$ & $5.0(1)$ & $6.19(1)$ & $5.8(1)$ \\
\hline$\theta /^{\circ}$ & +14.35 & +13.94 & +11.93 & +14.55 & $\pm 30.66(1)$ & $\pm 28.72(1)$ & $\pm 35.40(7)$ & $\pm 29.46(4)$ \\
\hline$\theta_{\mathrm{T}} /^{\circ}$ & +1.3 & - & - & - & +2.23 & - & - & - \\
\hline$\nu_{0} / \mathrm{cm}^{-1}$ & 34776 & - & - & - & $33038.55(10)$ & $33034.00(15)$ & $33039.87(19)$ & $33044.60(1)$ \\
\hline
\end{tabular}


a)



b)

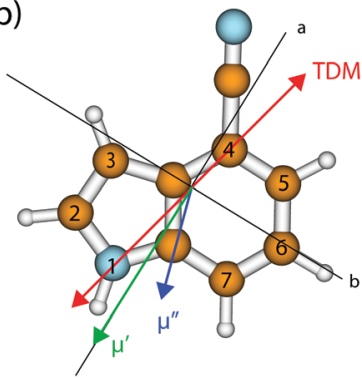

Fig. 1 (a) Definitions of the axis reorientation angle $\theta_{T}$, the angle of the transition dipole moment with the inertial $a$-axis $\theta$, and the angle of the permanent dipole moment with the inertial a-axis $\theta_{\mathrm{D}}$. The doubly primed axes refer to the ground state, the singly primed to the electronically excited state. A positive sign of $\theta_{\mathrm{T}}$ refers to a clockwise rotation of the inertial axis system upon electronic excitation, positive signs of $\theta$ and $\theta_{\mathrm{D}}$ refer to a clockwise rotation of the inertial a-axis onto the dipole/TDM vector. (b) Optimized ground state structure, atomic numbering, inertial axes, transition dipole moment for excitation to the lowest excited singlet state (red), and the orientation of the permanent dipole moment in the ground (blue) and first excited state (green) of 4-cyanoindole.

will change its geometry, leading to the consequence that these two geometries have to be described by two different molecule-fixed axis systems. They can be interconverted by a $3 \times 3$ rotational matrix, which is called the axis reorientation matrix $\neq$ For a molecule, which is planar in both electronic states a single axis reorientation angle $\theta_{\mathrm{T}}$ is needed to fully describe the axis reorientation.

From the SCS-CC2 optimized structures, the axis reorientation angle of the inertial axis system upon electronic excitation $\theta_{\mathrm{T}}$ can be determined using the relation for planar molecules given by Hougen and Watson: ${ }^{60}$

$$
\tan \left(\theta_{\mathrm{T}}\right)=\frac{\sum_{i} m_{i}\left(a_{i}^{\prime} b_{i}^{\prime \prime}-b_{i}^{\prime} a_{i}^{\prime \prime}\right)}{\sum_{i} m_{i}\left(a_{i}^{\prime} a_{i}^{\prime \prime}-b_{i}^{\prime} b_{i}^{\prime \prime}\right)}
$$

Here, the doubly primed coordinates refer to the principal axis system (PAS) in the electronic ground state and the singly primed quantities to the respective excited state inertial system and the $m_{i}$ are the atomic masses. Using the PAS coordinates of SCS-CC2 optimized structures for the ground and the lowest excited state, we obtain an axis reorientation angle $\theta_{\mathrm{T}}$ of $+1.3^{\circ}$. The positive sign of $\theta_{\mathrm{T}}$ refers to a clockwise rotation of the inertial axis system upon electronic excitation, $c f$. Fig. 1.

Another consequence of the inertial axis rotation is a rotational Duschinsky mixing of the vibrational modes of 4-cyanoindole. The vibrational modes of the electronically excited state can be expressed as linear combination of the ground state modes using the following linear orthogonal transformation, first given by Duchinsky: ${ }^{61}$

$$
Q_{i}^{\prime}=\sum_{j} S_{i j} Q_{j}^{\prime \prime}+d_{i}
$$

where $Q^{\prime}$ and $Q^{\prime \prime}$ are the $3 N-6$-dimensional vectors of the normal modes of excited and ground state, respectively, $S_{i j}$ is a

\# In the original publication the term axis switching was used instead of axis reorientation.

$3 N-6 \times 3 N-6$ rotation matrix (the Duchinsky matrix) and $d_{i}$ is an $3 N-6$-dimensional vector which contains the linear displacements along the normal coordinates.

The amount of mode mixing, which is contained in the Duschinsky matrix can easily be visualized graphically. Fig. 2 shows a representation the size of the elements in this matrix. Black squares refer to a matrix element of 1 , white squares of 0 , intermediate values are shown grey coded. Modes, which show no mixing are represented by a black square (not necessarily on the diagonal, because the order of the vibrations might interchange upon electronic excitation). Similar graphs for 3-cyanoindole and 5-cyanoindole are given in the ESI $\dagger$ (Fig. S1 and S2).

\subsection{Experimental results}

Fig. 3a shows the rotationally resolved electronic spectrum of the origin of 4-cyanoindole at $33038.55 \mathrm{~cm}^{-1}(0$ on the scale of the figure) along with the best fit at zero field (trace b) and at a field strength of $425.48 \mathrm{~V} \mathrm{~cm}^{-1}$ (trace c). The electric field in the chosen set-up is parallel to the polarization of the plane of the exciting light, thus $\Delta M=0$ selection rules for the Stark spectrum hold. The spectrum was fit using a CMA-ES; the molecular parameters from the fit are summarized in Table 1 and are compared to the results of the SCS-CC2/cc-pVTZ calculations. The spectrum is an $a b$-hybrid, which is mainly polarized along the $a$-axis.

The rotationally resolved spectra of the electronic origins of the 1-, 2- and 3-deuterated isotopologues at $33034.00 \mathrm{~cm}^{-1}$, $33039.87 \mathrm{~cm}^{-1}$ and $33044.60 \mathrm{~cm}^{-1}$ are shown in the ESI $\dagger$ (Fig. S4-S6). The molecular parameters, obtained from the CMA-ES fits are compiled in Table 1 . The angle $\theta$ of the TDM in 4-cyanoindole $\left(\mathrm{d}_{1}\right)$ is slightly smaller than in 4-cyanoindole.

3.2.1 Excited state lifetime. The excited state lifetime of 4-cyanoindole in the gas phase was obtained from the Lorentzian contribution of $14.4 \pm 0.2 \mathrm{MHz}$ to the Voigt line profile using a fixed Gaussian contribution of 15.2 MHz. The Gaussian contribution was determined from a Voigt fit to a few single rovibronic lines with the boundary condition of equal Gaussian contribution to each line. Fig. S3 of the ESI $\dagger$ shows an extended view of the experimental spectrum along with the best fit of the line profile parameters. The so determined lifetime is $\mathbf{1 1 . 0} \pm 0.2 \mathrm{~ns}$. This lifetime decreases to $5.0 \pm 0.1 \mathrm{~ns}$ upon N-deuteration and to 6.19 and $5.8 \mathrm{~ns}$ for deuteration in 2- and 3-position of 4-cyanoindole, respectively.

Additionally, we performed lifetime measurements of 3-cyanoindole, 4-cyanoindole, and 5-cyanoindole and indole in different solvents using TCSPC. Fig. 4 presents the decay curves for 4-cyanoindole and 4-cyanoindole $\left(\mathrm{d}_{1}\right)$ in ethyl acetate and $\mathrm{H}_{2} \mathrm{O}$ $\left(\mathrm{D}_{2} \mathrm{O}\right)$. The excited state lifetimes of isolated indole, 3-cyanoindole, 4-cyanoindole, and 5-cyanoindole and their N-deuterated isotopologues in ethyl acetate (EA) and $\mathrm{H}_{2} \mathrm{O}\left(\mathrm{D}_{2} \mathrm{O}\right.$ for the deuterated compounds) are summarized in Table 3.

\section{Discussion}

\subsection{Structural changes upon electronic excitation}

From the rotational constants of the normal and the 1-, 2- and 3-deuterated isotopologues, the position of the hydrogen atoms 
Table 2 SCS-CC2/cc-pVTZ computed vertical excitation energies, relative to the energy of the lowest excitation in $\mathrm{cm}^{-1}$, excited state dipole moment $\mu$ and oscillator strength $f$ of indole, 3-cyanoindole, 4-cyanoindole, and 5-cyanoindole. Negative values refer to interchanged order of $L_{a}$ and $L_{b}$ states





Fig. 2 Duschinsky matrix of the 45 vibrational modes of 4 -cyanoindole.



Fig. 3 Rotationally resolved electronic spectrum of the electronic origin of 4-cyanoindole, along with a simulation with the best CMA-ES fit parameters.

in 1-, 2- and 3-position of the pyrrole ring have been determined on the basis of Kraitchman's equations ${ }^{65}$ for planar asymmetric molecules in the ground and the electronically excited states, respectively. The $|a|$ and $|b|$ components of the Cartesian coordinates in the coordinate system of the reference isotopologue in the
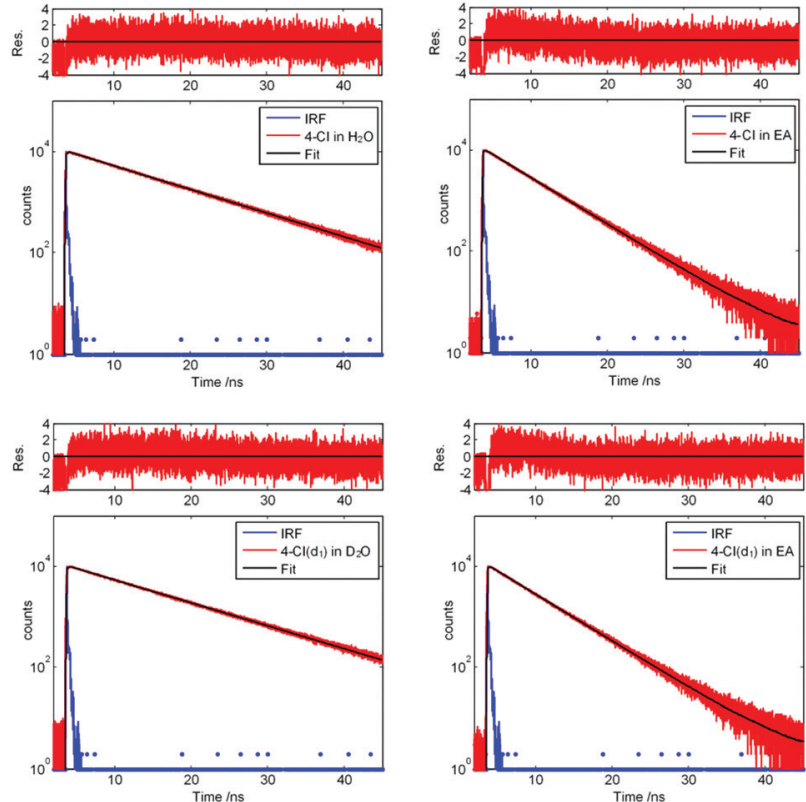

Fig. 4 TCSPC traces of 4-cyanoindole in $\mathrm{H}_{2} \mathrm{O}, 4$-cyanoindole in EA, 4-cyanoindole $\left(d_{1}\right)$ in $\mathrm{D}_{2} \mathrm{O}$, and 4-cyanoindole $\left(\mathrm{d}_{1}\right)$ in $\mathrm{EA}$, along with the instrument response function and the best fit to a single exponential decay.

electronic ground and excited states are given in Table 4 along with the center of mass (COM) distances of the respective atom.

\subsection{Permanent dipole moments}

The experimentally determined dipole moment increases from 6.31 D to $8.92 \mathrm{D}$ upon electronic excitation. This is a clear indication, that the excited state is of ${ }^{1} \mathrm{~L}_{\mathrm{a}}$ character. Such an increase of nearly $2 \mathrm{D}$ is not observed for excitation to ${ }^{1} \mathrm{~L}_{\mathrm{b}}$ states, in which the permanent dipole moment in general is equal or even smaller than in the electronic ground state. For 3-cyanoindole, for which the lowest state is of ${ }^{1} \mathrm{~L}_{\mathrm{b}}$ character, we found a decrease from $5.89 \mathrm{D}$ to $5.39 \mathrm{D}$ upon electronic excitation, ${ }^{40}$ while for 5 -cyanoindole (lowest excited singlet state is ${ }^{1} \mathrm{~L}_{\mathrm{a}}$ ) an increase of $1 \mathrm{D}$ is found. ${ }^{66}$ Thus, the change of permanent dipole moment upon excitation of the singlet state can be used as a sensitive indicator of the electronic nature of this excited state.

\subsection{Transition dipole moment and the electronic structure of the excited state}

The sign of the angle $\theta$ between the TDM and the $a$-axis cannot be determined directly from rotationally resolved spectra. 
Table 3 Angles $\theta$ of the orientation of the transition dipole moment and excited state lifetimes (molecular beam and solution) of indole (In), 3-cyanoindole $(3-\mathrm{Cl}), 4$-cyanoindole $(4-\mathrm{Cl}), 5$-cyanoindole $(5-\mathrm{Cl})$, the 3-cyanoindole-water cluster and the $\mathrm{N}$-deuterated isotopologues of 3-and 4-cyaynoindole $\left(\mathrm{d}_{1}\right)$. In contrast to the $\theta$ values presented in Table 1 , those shown here, are given with respect to the pseudo $C_{2}$-axis of indole

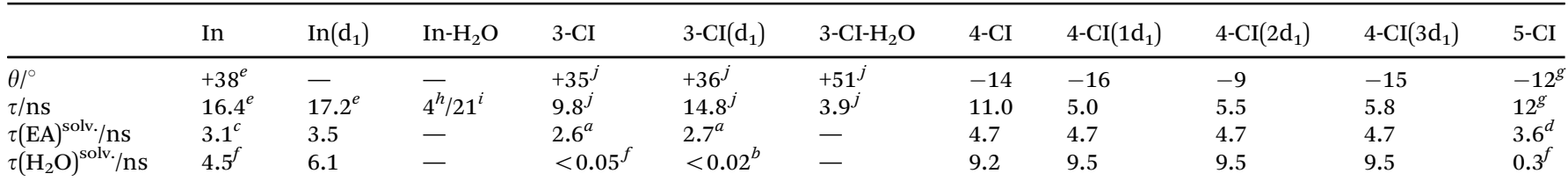

${ }^{a}$ Excitation of 3-cyanoindole and 3-cyanoindole $\left(\mathrm{d}_{1}\right)$ in ethyl acetate $(\mathrm{EA})$ at $283 \mathrm{~nm} .{ }^{b} 3$-CI $\left(\mathrm{d}_{1}\right)$ has been measured in $\mathrm{D}_{2} \mathrm{O}$ with excitation at $283 \mathrm{~nm}$. ${ }^{c}$ Excitation of indole in EA at $284 \mathrm{~nm} .{ }^{d}$ Excitation of 5-cyanoindole in EA at $295 \mathrm{~nm} .{ }^{e}$ Ref. $62 .{ }^{f}{\text { Ref. } 16 .{ }^{g}}$ Ref. $34 .{ }^{h}$ Ref. $63 .{ }^{i}$ Ref. $64 .{ }^{j}$ Ref. 40.

Table 4 Cartesian $r_{\mathrm{s}}$ coordinates and center of mass (COM) distances of the $\mathrm{H}$-atoms $1 \mathrm{a}, 2 \mathrm{a}$, and $3 \mathrm{a}$ of 4 -cyanoindole in pm from a Kraitchman analysis of the rotational constants of the respective isotopologues

\begin{tabular}{|c|c|c|c|c|c|c|}
\hline & \multicolumn{2}{|c|}{ 1-Position } & \multicolumn{2}{|c|}{ 2-Position } & \multicolumn{2}{|c|}{ 3-Position } \\
\hline & $|a|$ & $|b|$ & $|a|$ & $|b|$ & $|a|$ & $|b|$ \\
\hline $\mathrm{S}_{0} / \mathrm{pm}$ & 344.13 & 77.46 & 335.42 & 285.96 & 78.98 & 274.93 \\
\hline $\mathrm{COM} / \mathrm{pm}$ & 352.74 & & 440.77 & & 286.06 & \\
\hline $\mathrm{S}_{1} / \mathrm{pm}$ & 344.91 & 81.58 & 337.65 & 282.29 & 84.11 & 270.36 \\
\hline $\mathrm{COM} / \mathrm{pm}$ & 354.42 & & 440.11 & & 283.14 & \\
\hline
\end{tabular}

Only the projection of the TDM onto the main inertial axes is obtained from the intensities of the rovibronic transitions. However, there are two methods to obtain also the sign of the TDM angle. Pratt and coworkers have shown, that axis reorientation mixes the rotational wave functions of the ground and excited rovibronic state, and causes a quantum interference effect, which modulates the intensities of the rovibronic transitions. ${ }^{67}$ These interferences, which cause mixing of the wave functions and therefore generate anomalous intensities of hybrid bands can be considered in two ways. The original method of Hougen and Watson rotates the wave functions of the excited state into the coordinate system of the ground state after diagonalization of the respective Hamiltonian. ${ }^{60}$ Held $e t$ al. applied an equivalent but computationally faster method. They express the excited state Hamiltonian in the coordinate system of the ground state by means of a similarity transformation, i.e. they rotate the Hamiltonian prior to diagonalization. ${ }^{67}$ It has been shown that in the case of axis reorientation, the rovibronic line intensities depend on the relative sign of the axis reorientation angle $\theta_{\mathrm{T}}$ and the TDM angle $\theta$, which therefore can be determined experimentally. ${ }^{24,28,67,68}$

Fig. 5 shows a zoomed part of the fit of the rotationally resolved electronic spectrum of the electronic origin of 4-cyanoindole using an axis reorientation Hamiltonian and different combinations of $\theta_{\mathrm{T}}$ and $\theta$ signs. Obviously, the best agreement is obtained for $++(--)$ sign combination, while the fit using $+-(-+)$ combinations has larger intensity deviations. Equally, a fit without axis reorientation $\left(\theta_{\mathrm{T}}=0\right)$ is inferior with respect to the agreement between experimental and simulated intensities.

Since the $a b$ initio calculations yielded a positive value of $\theta_{\mathrm{T}}$ (cf. Section 3.1), the sign of $\theta$ must also be positive. This means that the rotation of the $a$-axis onto the TDM is clockwise, as shown in Fig. 1. This orientation of the TDM belong to an $\mathrm{L}_{\mathrm{a}}$ state.

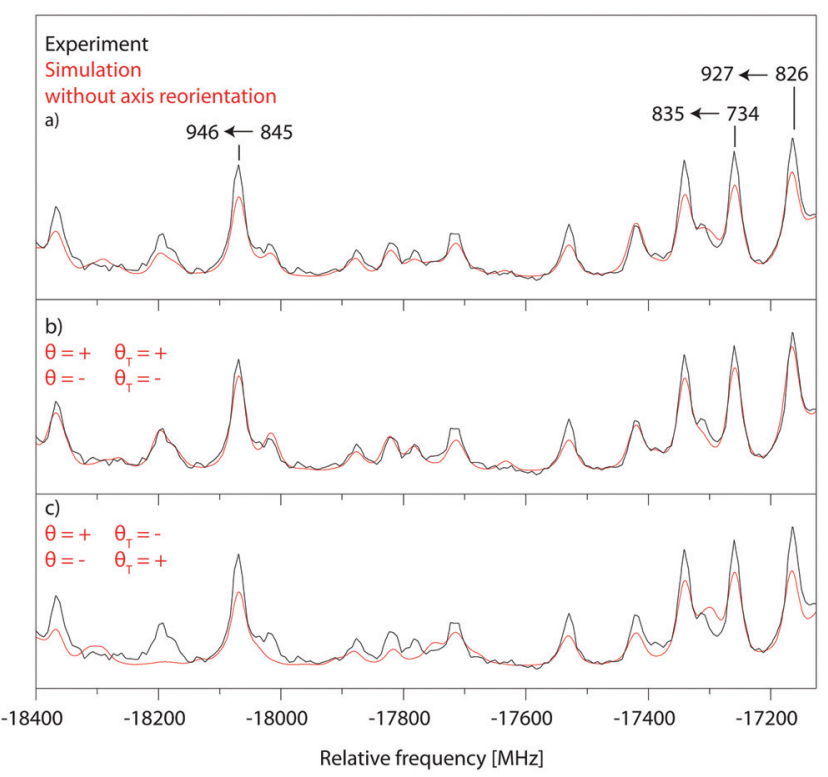

Fig. 5 Comparison of a zoomed part of the P-branch of the electronic origin spectrum of 4-cyanoindole, with fits using an axis reorientation Hamiltonian. (a) Experiment vs. fit without axis reorientation $\left(\theta_{\mathrm{T}}=0\right)$. (b) Experiment vs. fit with axis reorientation $\left(\theta=+\right.$ and $\theta_{\mathrm{T}}=+$ or $\theta=-$ and $\theta_{\mathrm{T}}=-$ ). (c) Experiment $v$ s. fit with axis reorientation $\left(\theta=+\right.$ and $\theta_{\mathrm{T}}=-\operatorname{or} \theta=-$ and $\theta_{\mathrm{T}}=+$ ).

Independently, the orientation of the TDM can be obtained from the comparison of the spectra of the normal and the 2- and 3-deuterated isotopologues. ${ }^{69}$ Deuteration rotates the inertial $a$-axis clockwise, as can be seen from Fig. 1. If the rotation of the $a$-axis onto the TDM is also clockwise (positive), the angle $\theta$ will become smaller upon $\mathrm{N}$-deuteration, otherwise it will become larger. Table 1 shows that $\theta$ decreases from $30.7^{\circ}$ to $28.7^{\circ}$, confirming the positive sign of $\theta$ from the axis reorientation.

In order to facilitate the comparison of TDM orientations between the different conformers, the angle of the TDM is given with respect to the pseudo- $C_{2}$ axis of indole, $\S$ in spite of the inertial $a$-axis, which will rotate with the change of the substitution position. These projections have been made in Table 3. Clearly, indole $\left(+38^{\circ}\right)$ and 3-cyanoindole $\left(+35^{\circ}\right)$ have similar TDM orientations,

$\S$ This axis is close to the inertial $a$-axis in indole. It would be of exact $C_{2}$ symmetry axis in the hydrocarbon indane. Since the $\mathrm{L}_{\mathrm{a}}$ and $\mathrm{L}_{\mathrm{b}}$ nomenclature of Platt refers to the TDM vector intersecting the atoms or bonds, it is convenient to use this pseudo- $C_{2}$ axis of indole for a better comparison. 


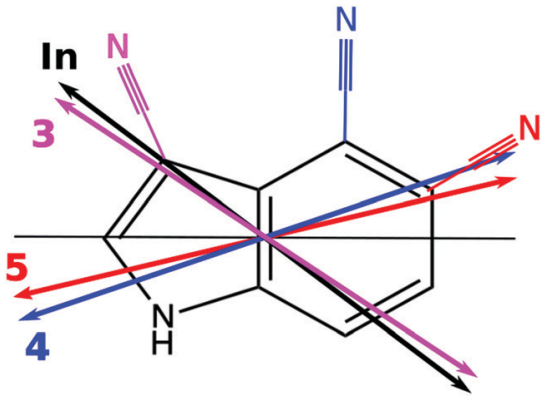

Fig. 6 Orientation of the TDM of indole (black double arrow), 3-cyanoindole (magenta double arrow), 4-cyanoindole (blue double arrow), and 5 -cyanoindole (red double arrow), relative to the pseudo- $C_{2}$ axis of indole (black line).

belonging to an ${ }^{1} \mathrm{~L}_{\mathrm{b}}$ state, while 4 -cyanoindole $\left(-14^{\circ}\right)$ and 5 -cyanoindole $\left(-12^{\circ}\right)$ among each other have similar ${ }^{1} \mathrm{~L}_{\mathrm{b}}$ like TDM orientations (Fig. 6).

What is now the reason that the lowest electronically excited state in 4- and 5-cyanoindole is of ${ }^{1} \mathrm{~L}_{\mathrm{a}}$ character, while it is ${ }^{1} \mathrm{~L}_{\mathrm{b}}$ like in 3-cyanoindole? Looking to the electron density difference plots upon $S_{1} \leftarrow S_{0}$ excitation, shown in the ESI $\dagger$ (Fig. S8) reveals, that only in 4- and 5-cyanoindole the electron density at the cyano group changes upon excitation.

\subsection{Excited state lifetimes}

4.4.1 Gas phase. For isolated 4-cyanoindole, the experimentally determined lifetime of $\mathbf{1 1 . 0} \mathrm{ns}$ is nearly twice as large as for the deuterated species. The lifetime of the three investigated deuterated 4-cyanoindoles is remarkably independent on the position of deuteration. N(1)-D, C(2)-D, and $\mathrm{C}(3)-\mathrm{D}$ have lifetimes of 5.0, 6.19 and $5.8 \mathrm{~ns}$, respectively. Mostly, the excited state lifetimes of deuterated species are longer than those of the undeuterated ones. A few examples of systems, for which information about the lifetime of the isolated species upon $\mathrm{N}(\mathrm{H})$-deuteration, $\mathrm{O}(\mathrm{H})$-deuteration and $\mathrm{C}(\mathrm{H})$-deuteration are available will be discussed in the following.

For indole, the chromophore of 4-cyanoindole, the lifetime increases slightly from 16.4 to $17.2 \mathrm{~ns}$ upon $\mathrm{N}(\mathrm{H})$-deuteration. ${ }^{62}$ In isolated 3-cyanoindole an increase from $9.8 \mathrm{~ns}$ to $14.8 \mathrm{~ns}$ is observed. ${ }^{40}$ For aniline it has been found that the excited state lifetime of $8.51 \mathrm{~ns}$ decreases to $8.48 \mathrm{~ns}$ upon $\mathrm{N}-\mathrm{d}_{2}$ substitution, but increases to $9.13 \mathrm{~ns}$ upon $\mathrm{C}-\mathrm{d}_{5}$ substitution. ${ }^{70}$

In phenol, the effect is more pronounced: OH-deuteration increases the excited state lifetime from $2.4 \mathrm{~ns}$ to $13.3 \mathrm{~ns}$. The effect of C-deuteration on the lifetime strongly depends on the position of deuteration. Deuteration in para-position with respect to the hydroxy group leads to a lifetime of $2.1 \mathrm{~ns}$, while combined $\mathrm{O}(\mathrm{H})$-deuteration in ortho- or meta-position leads to substantially higher lifetimes above $30 \mathrm{~ns}^{71}$

In any case, deuteration (CD, ND, and OD) at the chromophore leads in the majority of cases to an increase of the excited state lifetime, in contrast to the findings for 4-cyanoindole. Even in the chemically similar 3-cyanoindole a $50 \%$ increase of excited state lifetime was observed. The reason for the obvious deviation in the case of 4-cyanoindole is subject to further investigation.
4.4.2 Solution. The lifetimes of 4-cyanoindole and 4-cyanoindole $\left(\mathrm{d}_{1}\right)$ in ethyl acetate are in general much shorter, than those, measured in $\mathrm{H}_{2} \mathrm{O}$ or $\mathrm{D}_{2} \mathrm{O}$. The effect of changing the solvent is thus much larger, than deuteration of the chromophore. Due to isotopic scrambling, we cannot make a definite assignment to a specific deuteration position of 4-cyanoindole in $\mathrm{D}_{2} \mathrm{O}$. Independent on the deuteration rate, a single exponential decay is found, with a lifetime of 4.7 ns. Polar solvents are known to increase the excited state lifetime, when the ground state of the solute is stabilized more than the excited state, thus increasing the energy gap. ${ }^{72}$ This explanation does not hold in this case, since the dipole moment of water $(1.85 \mathrm{D})$ and of ethyl acetate $(1.78 \mathrm{D})$ are very similar, and the excited state dipole moment of 4-cyanoindole is larger than its ground state dipole. Burghardt and Hynes have shown that solvent molecules might act as an additional tuning mode which modulates the energy gap and displaces the CI from its vacuum value. ${ }^{73}$

\subsection{Fluorescence quantum yields}

The pure radiative lifetime of a transition from initial state i to final state $\mathrm{f}$ can be approximated by:

$$
\tau_{\text {nat }}=\frac{c^{3}}{8 \pi h \nu_{\mathrm{fi}}{ }^{3}} \frac{6 \varepsilon_{0} \hbar^{2}}{\left|\mu_{\mathrm{fi}}\right|^{2}}
$$

where $\nu_{\mathrm{fi}}$ is the transition frequency and $\left|\mu_{\mathrm{fi}}\right|^{2}$ the dipole strength (in $\mathrm{D}^{2}$ ) for this transition. The dipole strength can be calculated from the dimensionless oscillator strength $f$ :

$$
\left|\mu_{\mathrm{fi}}\right|^{2}=\frac{f h e^{2}}{8 \pi m_{\mathrm{e}} \nu_{\mathrm{fi}}}
$$

The ratio of the experimental lifetime $\tau_{\exp }$, which is the inverse of the sum of all radiative and the nonradiative deactivation paths and the natural life time $\tau_{\text {nat }}$, as the inverse of the fluorescence rate constant $k_{\mathrm{f}}$ yields the fluorescence quantum yield:

$$
Q=\frac{k_{\mathrm{f}}}{k_{\mathrm{f}}+\sum k_{\mathrm{nr}}}=\frac{k_{\mathrm{f}}}{\sum k_{i}}=\frac{\tau_{\exp }}{\tau_{\mathrm{nat}}}
$$

Using the experimental transition frequencies and lifetimes from Table 1 and the dimensionless oscillator strengths $f$ from the $a b$ initio calculations given in Table 2, the fluorescence quantum yield of isolated 4-cyanoindole is calculated to be 0.42 . For 3-cyanoindole a similar value of 0.43 has been found using the parameters from ref. 40, while for 5-cycanoindole a substantially smaller quantum yield of 0.10 is found with the parameters from ref. 34. In aqueous solution, that quantum yield of 4-cyanoindole has been determined to 0.85 at an excitation wavelength of $270 \mathrm{~nm} .{ }^{17}$

\section{Conclusions}

The lowest electronically excited singlet state of 4-cyanoindole is the ${ }^{1} \mathrm{~L}_{\mathrm{a}}$ state, as in the case of 5 -cyanoindole, but contrary to 3-cyanoindole. This assignment has been made from the experimentally determined orientations of the transition dipole moment as well as the orientations and absolute values of the 
permanent dipole moments in the ground and excited state. Also the fluorescence lifetime could be extracted from the Lorentz contribution to the Voigt profile of the rovibronic lines. Thus, rotationally resolved electronic Stark spectroscopy is not only capable of determining structural parameters of electronically excited states, but also to assign the electronic nature of the excited state and obtain information about dynamical processes in the excited state.

\section{Conflicts of interest}

There are no conflicts to declare.

\section{Acknowledgements}

Financial support of the Deutsche Forschungsgemeinschaft via grant SCHM1043/12-3 is gratefully acknowledged. Computational support and infrastructure was provided by the "Center for Information and Media Technology" (ZIM) at the HeinrichHeine-University Düsseldorf (Germany).

\section{References}

1 P. R. Callis, Methods Enzymol., 1997, 278, 113-150.

2 A. L. Sobolewski, W. Domcke, C. Dedonder-Lardeux and C. Jouvet, Phys. Chem. Chem. Phys., 2002, 4, 1093-1100.

3 P. R. Callis, Methods Enzymol., 2011, 487, 1-38.

4 W. Domcke, D. Yarkony and H. Köppel, Conical Intersections: Electronic Structure, Dynamics \& Spectroscopy, World Scientific Publishing, Singapore, 2004.

5 D. G. Truhlar and C. Mead, Phys. Rev. A: At., Mol., Opt. Phys., 2003, 68, 032501.

6 A. L. Sobolewski and W. Domcke, J. Phys. Chem. A, 2001, 105, 9275-9283.

7 A. L. Sobolewski and W. Domcke, Chem. Phys. Lett., 1999, 315, 293-298.

8 A. L. Sobolewski and W. Domcke, Eur. Phys. J. D, 2002, 20, 369-374.

9 P. R. Callis, J. Chem. Phys., 1991, 95, 4230.

10 C. Brand, J. Küpper, D. W. Pratt, W. L. Meerts, D. Krügler, J. Tatchen and M. Schmitt, Phys. Chem. Chem. Phys., 2010, 12, 4968-4997.

11 P. R. Callis, J. T. Vivian and L. S. Slater, Chem. Phys. Lett., 1995, 244, 53.

12 L. S. Slater and P. R. Callis, J. Phys. Chem., 1995, 99, 8572.

13 M. Böhm, J. Tatchen, D. Krügler, K. Kleinermanns, M. G. D. Nix, T. A. LeGreve, T. S. Zwier and M. Schmitt, J. Phys. Chem. A, 2009, 113, 2456-2466.

14 L. J. G. W. van Wilderen, H. Brunst, H. Gustmann, J. Wachtveitl, J. Broosc and J. Bredenbeck, Phys. Chem. Chem. Phys., 2018, 20, 19906-19915.

15 B. N. Markiewicz, D. Mukherjee, T. Troxler and F. Gai, J. Phys. Chem. B, 2016, 210, 936-944.

16 M. R. Hilaire, D. Mukherjee, T. Troxler and F. Gai, Chem. Phys. Lett., 2017, 685, 133-138.
17 M. R. Hilaire, I. A. Ahmed, C.-W. Lin, H. Jo, W. F. DeGrado and F. Gai, Proc. Natl. Acad. Sci. U. S. A., 2017, 114, 6005-6009.

18 J. R. Platt, J. Chem. Phys., 1949, 17, 484-495.

19 G. Weber, Biochem. J., 1960, 75, 335-345.

20 B. Albinsson and B. Nordén, J. Phys. Chem., 1992, 96, 6204.

21 D. M. Sammeth, S. Yan, L. H. Spangler and P. R. Callis, J. Phys. Chem., 1990, 94, 7340.

22 T. L. O. Barstis, L. I. Grace, T. M. Dunn and D. L. Lubman, J. Phys. Chem., 1993, 97, 5820.

23 L. A. Philips and D. H. Levy, J. Chem. Phys., 1986, 85, 1327-1332.

24 G. Berden, W. L. Meerts and E. Jalviste, J. Chem. Phys., 1995, 103, 9596-9606.

25 C. Kang, T. M. Korter and D. W. Pratt, J. Chem. Phys., 2005, 122, 174301.

26 J. Zuclich, J. U. von Schütz and A. H. Maki, J. Am. Chem. Soc., 1974, 96, 710-714.

27 B. J. Fender, K. W. Short, D. K. Hahn and P. R. Callis, Int. J. Quantum Chem., 1999, 72, 347-356.

28 J. Küpper, D. W. Pratt, W. L. Meerts, C. Brand, J. Tatchen and M. Schmitt, Phys. Chem. Chem. Phys., 2010, 12, 4980-4988.

29 L. Serrano-Andrés and B. O. Roos, J. Am. Chem. Soc., 1996, 118, 185-195.

30 A. C. Borin and L. Serrano-Andrés, Chem. Phys., 2000, 262, 253-265.

31 L. Serrano-Andrés and A. C. Borin, Chem. Phys., 2000, 262, 267-283.

32 X. Meng, T. Harricharran and L. J. Juszczak, Photochem. Photobiol., 2013, 89, 40-50.

33 W. Domcke and A. L. Sobolewski, Science, 2003, 302, 1693-1694.

34 O. Oeltermann, C. Brand, B. Engels, J. Tatchen and M. Schmitt, Phys. Chem. Chem. Phys., 2012, 14, 10266-10270.

35 C. Brand, B. Happe, O. Oeltermann, M. Wilke and M. Schmitt, J. Mol. Struct., 2013, 1044, 21-25.

36 B. Stuhlmann, A. Gräßle and M. Schmitt, Phys. Chem. Chem. Phys., 2014, 16, 899-905.

37 A. Min, C. J. Moon, A. Ahn, J. H. Lee, S. K. Kim and M. Y. Choi, Chem. Phys. Lett., 2016, 658, 63-70.

38 A. Ahn, A. Min, C. J. Moon, J. H. Lee and M. Y. Choi, Chem. Phys. Lett., 2014, 616-617, 55-60.

39 A. Min, A. Ahn, C. J. Moon, J. H. Lee, M. Y. Choi and S. K. Kim, Chem. Phys. Lett., 2014, 614, 263-268.

40 M. Schneider, M.-L. Hebestreit, M. M. Lindic, H. Parsian, A. Y. Torres-Boy, L. Álvarez Valtierra, L. Meerts, R. Kühnemuth and M. Schmitt, Phys. Chem. Chem. Phys., 2018, 20, 23441-23452.

41 Y. Huang and M. Sulkes, Chem. Phys. Lett., 1996, 254, 242-248.

42 S. Gerstenkorn and P. Luc, Atlas du spectre d'absorption de la molécule d'iode 14800-20000 $\mathrm{cm}^{-1}$, CNRS, Paris, 1986.

43 M. Schmitt, Habilitation, Heinrich-Heine-Universität, Math. Nat. Fakultät, Düsseldorf, 2000.

44 M. Schmitt, J. Küpper, D. Spangenberg and A. Westphal, Chem. Phys., 2000, 254, 349-361.

45 J. Wilke, M. Wilke, W. L. Meerts and M. Schmitt, J. Chem. Phys., 2016, 144, 044201.

46 K. Wohlfart, M. Schnell, J. U. Grabow and J. Küpper, J. Mol. Spectrosc., 2014, 247, 119-121. 
47 R. Ahlrichs, M. Bär, M. Häser, H. Horn and C. Kölmel, Chem. Phys. Lett., 1989, 162, 165-169.

48 J. T. H. Dunning, J. Chem. Phys., 1989, 90, 1007-1023.

49 C. Hättig and F. Weigend, J. Chem. Phys., 2000, 113, 5154-5161.

50 C. Hättig and A. Köhn, J. Chem. Phys., 2002, 117, 6939-6951.

51 C. Hättig, J. Chem. Phys., 2002, 118, 7751-7761.

52 A. Hellweg, S. Grün and C. Hättig, Phys. Chem. Chem. Phys., 2008, 10, 1159-1169.

53 P. Deglmann, F. Furche and R. Ahlrichs, Chem. Phys. Lett., 2002, 362, 511-518.

54 W. L. Meerts, M. Schmitt and G. Groenenboom, Can. J. Chem., 2004, 82, 804-819.

55 W. L. Meerts and M. Schmitt, Phys. Scr., 2005, 73, C47-C52.

56 W. L. Meerts and M. Schmitt, Int. Rev. Phys. Chem., 2006, 25, 353-406.

57 M. Schmitt and W. L. Meerts, in Handbook of High Resolution Spectroscopy, ed. M. Quack and F. Merkt, John Wiley and Sons, 2011.

58 A. Ostenmeier, A. Gawelcyk and N. Hansen, in Parallel Problem Solving from Nature, PPSN III, ed. Y. Davidor, H.-P. Schwefel and R. Männer, Springer, Berlin/Heidelberg, 1994. 59 N. Hansen and A. Ostermeier, Evol. Comput., 2001, 9, 159-195.

60 J. T. Hougen and J. K. G. Watson, Can. J. Phys., 1965, 43, 298-320.
61 F. Duschinsky, Acta Physicochim. URSS, 1937, 7, 551-577.

62 G. A. Bickel, D. R. Demmer, E. A. Outhouse and S. C. Wallace, J. Chem. Phys., 1989, 91, 6013.

63 T. M. Korter, D. W. Pratt and J. Küpper, J. Phys. Chem. A, 1998, 102, 7211-7216.

64 S. Arnold and M. Sulkes, J. Phys. Chem., 1992, 96, 4768.

65 J. Kraitchman, Am. J. Phys., 1953, 21, 17.

66 J. Wilke, M. Wilke, C. Brand, W. L. Meerts and M. Schmitt, ChemPhysChem, 2016, 17, 2736-2743.

67 A. Held, B. B. Champagne and D. W. Pratt, J. Chem. Phys., 1991, 95, 8732 .

68 C. Brand, O. Oeltermann, M. Wilke, J. Tatchen and M. Schmitt, ChemPhysChem, 2012, 13, 3134-3138.

69 M. Schmitt, D. Krügler, M. Böhm, C. Ratzer, V. Bednarska, I. Kalkman and W. L. Meerts, Phys. Chem. Chem. Phys., 2006, 8, 228-235.

70 R. Scheps, D. Florida and S. A. Rice, J. Chem. Phys., 1974, 61, 1730-1747.

71 C. Ratzer, J. Küpper, D. Spangenberg and M. Schmitt, Chem. Phys., 2002, 283, 153-169.

72 A. Kahan, A. Wand, S. Ruhman, S. Zilberg and Y. Haas, J. Phys. Chem. A, 2011, 115, 10854-10861.

73 I. Burghardt and J. T. Hynes, J. Phys. Chem. A, 2006, 115, 11411-11423. 